\title{
New perspectives in Arakelov geometry
}

\author{
Caterina Consani and Matilde Marcolli
}

\begin{abstract}
In this paper we give a unified description of the archimedean and the totally split degenerate fibers of an arithmetic surface, using operator algebras and Connes' theory of spectral triples in noncommutative geometry.
\end{abstract}

\section{Introduction.}

The aim of this article is to report on some of the recent results obtained by the two authors on a non-commutative interpretation of the totally split degenerate fibers of an arithmetic surface. The results stated in the first part of the paper were announced in a talk given by the first author at the VII meeting of the CNTA in May 2002 at Montreal. The material presented in this note is based on the papers [11] and [12].

Let $\mathcal{X}$ be an arithmetic surface defined over $\operatorname{Spec}(\mathbb{Z})$ (or over $\operatorname{Spec}\left(\mathcal{O}_{\mathbb{K}}\right)$, for a number field $\mathbb{K}$ ), having the smooth algebraic curve $X_{/ \mathbb{Q}}$ as its generic fiber. It is well known that, as a Riemann surface, $X_{/ \mathbb{C}}$ admits always a uniformization by means of a Schottky group $\Gamma$. In fact, the presence of this uniformization plays a fundamental role in the theory of the "fiber at infinity" of $\mathcal{X}$ described in [22] and [11]. In analogy to Mumford's p-adic uniformization of algebraic curves ( $c f$. [26), the Riemann surface $X_{/ \mathbb{C}}$ can be interpreted as the boundary at infinity of a 3-manifold $\mathfrak{X}_{\Gamma}$ defined as the quotient of the real hyperbolic 3 -space $\mathbb{H}^{3}$ by the action of the Schottky group $\Gamma$. The space $\mathfrak{X}_{\Gamma}$ contains in its interior an infinite link of bounded geodesics. Manin gave an expression for the Arakelov Green function on $X_{/ \mathbb{C}}$ in terms of configurations of geodesics in $\mathfrak{X}_{\Gamma}$, thus interpreting this tangle as the dual graph $\mathcal{G}$ of the "closed fiber at infinity" of $\mathcal{X}$.

In the first part of this paper we concentrate on Manin's description of such dual graph and we exhibit the suspension flow $\mathcal{S}_{T}$ of a dynamical system $T$ on the limit set of the Schottky group $\Gamma$ as a geometric model for the dual graph $\mathcal{G}$. In particular, the first cohomology group of $\mathcal{S}_{T}$ determines a model of the first cohomology of the dual graph of the "fiber at infinity". Furthermore, the first (co)homology group of $\mathcal{S}_{T}$ carries a natural filtration.

A crucial feature of this construction is the fact (proved in $\S 5$ of [11]) that this dynamical cohomology contains a subspace isomorphic to the Archimedean cohomology of [10]: the group of invariants for the action of the local monodromy "at infinity". This space has also the correct geometric properties, in order to be interpreted in terms of a cohomology theory associated to a degeneration in a "neighborhood of arithmetic infinity". Our result identifies such space with a distinguished subspace of the cohomology of a topological space constructed in terms of geodesics in $\mathfrak{X}_{\Gamma}$, our geometrc model of the dual graph. Under this identification, the graded structure associated to the filtration on the (co)homology of $\mathcal{S}_{T}$ corresponds to the graded structure given by Tate twists on the Archimedean cohomology of 10 .

The Cuntz-Krieger algebra $\mathcal{O}_{A}$ associated to the shift $T$ describes the "ring of functions" on a noncommutative space, which represents the quotient of the limit set $\Lambda_{\Gamma}$ of the Schottky group, by the action of $\Gamma$. In terms of the geometry of the fiber at arithmetic infinity, this space can be thought of as the set of components of the special fiber, or equivalently the vertices of the dual graph $\mathcal{G}$, 
whereas the quotient $\Lambda_{\Gamma} \times_{\Gamma} \Lambda_{\Gamma}$ gives the edges of $\mathcal{G}$. The algebra $\mathcal{O}_{A}$ carries a refined information on the action of the Schottky group $\Gamma$ on its limit set. In particular, we construct a spectral triple for this algebra.

In non-commutative geometry, the notion of a spectral triple provides the correct generalization of the classical structure of a Riemannian manifold. The two notions agree on a commutative space. In the usual context of Riemannian geometry, the definition of the infinitesimal element $d s$ on a smooth spin manifold can be expressed in terms of the inverse of the classical Dirac operator $D$. This is the key remark that motivates the theory of spectral triples. In particular, the geodesic distance between two points on the manifold is defined in terms of $D^{-1}(c f .8[\S \mathrm{VI})$. The spectral triple that describes a classical Riemannian spin manifold is $(A, H, D)$, where $A$ is the algebra of complex valued smooth functions on the manifold, $H$ is the Hilbert space of square integrable spinor sections, and $D$ is the classical Dirac operator (a square root of the Laplacian). These data determine completely and uniquely the Riemannian geometry on the manifold. It turns out that, when expressed in this form, the notion of spectral triple extends to more general non-commutative spaces, where the data $(A, H, D)$ consist of a $\mathrm{C}^{*}$-algebra $A$ (or more generally of a smooth subalgebra of a $\mathrm{C}^{*}$-algebra) with a representation as bounded operators on a Hilbert space $H$, and an operator $D$ on $H$ that verifies the main properties of a Dirac operator. The notion of smoothness is determined by $D$ : the smooth elements of $A$ are defined by the intersection of domains of powers of the derivation given by commutator with $|D|$. The basic geometric structure encoded by the theory of spectral triples is Riemannian geometry, but in more refined cases, such as Kähler geometry, the additional structure can be easily encoded as additional symmetries.

In these constructions, the Dirac operator $D$ is obtained from the grading operator associated to a filtration on the cochains of the complex that computes the dynamical cohomology. The induced operator on the subspace identified with the Archimedean cohomology agrees with the "logarithm of Frobenius" of [10] and [15].

This structure further enriches the geometric interpretation of the Archimedean cohomology, giving it the meaning of spinors on a noncommutative manifold, with the logarithm of Frobenius introduced in [15] in the role of the Dirac operator.

An advantage of this construction is that a completely analogous formulation exists in the case of Mumford curves. This provides a unified description of the archimedean and totally split degenerate fibers of an arithmetic surface.

Let $p$ be a finite prime where $\mathcal{X}$ has totally split degenerate reduction. Then, the completion $\hat{X}_{p}$ at $p$ of the generic fiber of $\mathfrak{X}$ is a split-degenerate stable curve over $\mathbb{Q}_{p}$ (also called a Mumford curve) uniformized by the action of a $p$-adic Schottky group $\Gamma$. The dual graph of the reduction of $\hat{X}_{p}$ coincides with a finite graph obtained as the quotient of a tree $\Delta_{\Gamma}$ by the action of $\Gamma$.

The curve $\hat{X}_{p}$ is holomorphically isomorphic to a quotient of a subset of the ends of the BruhatTits tree $\Delta$ of $\mathbb{Q}_{p}$ by the action of $\Gamma$. Thus, in this setting, the Bruhat-Tits tree at $p$ replaces the hyperbolic space $\mathbb{H}^{3}$ "at infinity", and the analog of the tangle of bounded geodesics in $\mathfrak{X}_{\Gamma}$ is played by doubly infinite walks in $\Delta_{\Gamma} / \Gamma$.

In analogy with the archimedean construction, we define the system $\left(\mathcal{W}\left(\Delta_{\Gamma} / \Gamma\right), T\right)$ where $T$ is an invertible shift map on the set $\mathcal{W}\left(\Delta_{\Gamma} / \Gamma\right)$ of doubly-infinite walks on the graph $\Delta_{\Gamma} / \Gamma$. The first cohomology group $H^{1}\left(\mathcal{W}\left(\Delta_{\Gamma} / \Gamma\right)_{T}, \mathbb{Z}\right)$ of the mapping torus $\mathcal{W}\left(\Delta_{\Gamma} / \Gamma\right)_{T}$ of $T$ inherits a natural filtration using which we introduce a dynamical cohomology group. We again have a Cuntz-Krieger graph algebra $C^{*}\left(\Delta_{\Gamma} / \Gamma\right)$ and we can construct a spectral triple as in the case at infinity, where again the Dirac operator is related to the grading operator $\Phi$ that computes the local factor as a regularized determinant, as in [16, 17. In [12, we also suggested a possible way of extending such construction to places that are not of split degenerate reduction, inspired by the "foam space" construction of [21] and 5 .

Acknowledgment. This paper was partly written during visits of the second author to Florida State University and University of Toronto. We thank these institutions for the hospitality. The first author is partially supported by NSERC grant 72016789. The second author is partially supported by the 
Humboldt Foundation and the German Government (Sofja Kovalevskaya Award). We thank Alain Connes for many extremely helpful discussions.

\section{Notation.}

Throughout this paper we will denote by $K$ one among the following fields: (a) the complex numbers $\mathbb{C}$, (b) a finite extension of $\mathbb{Q}_{p}$. When (b) occurs, we write $\mathcal{O}_{K}$ for the ring of integers of $K, \mathfrak{m} \subset \mathcal{O}_{K}$ for the maximal ideal and $\pi \in \mathfrak{m}$ for a uniformizer (i.e. $\mathfrak{m}=(\pi)$ ). We also denote by $k$ the residue classes field $k=\mathcal{O} / \mathfrak{m}$.

We denote by $\mathbb{H}^{3}$ the three-dimensional real hyperbolic space i.e. the quotient

$$
\mathbb{H}^{3}=\mathrm{SU}(2) \backslash \mathrm{PGL}(2, \mathbb{C}) .
$$

This space can also be described as the upper half space $\mathbb{H}^{3} \simeq \mathbb{C} \times \mathbb{R}^{+}$endowed with the hyperbolic metric. The group $\operatorname{PSL}(2, \mathbb{C})$ acts on $\mathbb{H}^{3}$ by isometries. The complex projective line $\mathbb{P}^{1}(\mathbb{C})$ is identified with the conformal boundary at infinity of $\mathbb{H}^{3}$ and the action of $\operatorname{PSL}(2, \mathbb{C})$ on $\mathbb{H}^{3}$ extends to an action on $\overline{\mathbb{H}}^{3}:=\mathbb{H}^{3} \cup \mathbb{P}^{1}(\mathbb{C})$. The group $\operatorname{PSL}(2, \mathbb{C})$ acts on $\mathbb{P}^{1}(\mathbb{C})$ by fractional linear transformations.

For an integer $g \geq 1$, a Schottky group of rank $g$ is a discrete subgroup $\Gamma \subset \operatorname{PSL}(2, K)$, which is purely loxodromic and isomorphic to a free group of rank $g$. We denote by $\Lambda_{\Gamma}$ the limit set of the action of $\Gamma$. One sees that $\Lambda_{\Gamma}$ is contained in $\mathbb{P}^{1}(K)$. This set can also be described as the closure of the set of the attractive and repelling fixed points $z^{ \pm}(g)$ of the loxodromic elements $g \in \Gamma$. In the case $g=1$ the limit set consists of two points, but for $g \geq 2$ the limit set is usually a fractal of some Hausdorff dimension $0 \leq \delta=\operatorname{dim}_{H}\left(\Lambda_{\Gamma}\right)<2$. We denote by $\Omega_{\Gamma}=\Omega_{\Gamma}(K)$ the domain of discontinuity of $\Gamma$, that is, the complement of $\Lambda_{\Gamma}$ in $\mathbb{P}^{1}(K)$.

When $K=\mathbb{C}$, the quotient space $\mathfrak{X}_{\Gamma}:=\mathbb{H}^{3} / \Gamma$ is topologically a handlebody of genus $g$, and the quotient $X_{/ \mathbb{C}}=\Omega_{\Gamma} / \Gamma$ is a Riemann surface of genus $g$. The covering $\Omega_{\Gamma} \rightarrow X_{/ \mathbb{C}}$ is called a Schottky uniformization of $X_{/ \mathbb{C}}$. Every complex Riemann surface $X_{/ \mathbb{C}}$ admits a Schottky uniformization. The handlebody $\mathfrak{X}_{\Gamma}$ can be compactified by adding the conformal boundary at infinity $X_{/ \mathbb{C}}$ to obtain $\overline{\mathfrak{X}}_{\Gamma}:=\mathfrak{X}_{\Gamma} \cup X_{/ \mathbb{C}}=\left(\mathbb{H}^{3} \cup \Omega_{\Gamma}\right) / \Gamma$.

A directed graph $E$ consists of data $E=\left(E^{0}, E^{1}, E_{+}^{1}, r, s, \iota\right)$, where $E^{0}$ is the set of vertices, $E^{1}$ is the set of oriented edges $w=\{e, \epsilon\}$, where $e$ is an edge of the graph and $\epsilon= \pm 1$ is a choice of orientation. The set $E_{+}^{1}$ consists of a choice of orientation for each edge, namely one element in each pair $\{e, \pm 1\}$. The maps $r, s: E^{1} \rightarrow E^{0}$ are the range and source maps, and $\iota$ is the involution on $E^{1}$ defined by $\iota(w)=\{e,-\epsilon\}$.

A directed graph is finite if $E^{0}$ and $E^{1}$ are finite sets. It is locally finite if each vertex emits and receives at most finitely many oriented edges in $E^{1}$. A vertex $v$ in a directed graph is a sink if there is no edge in $E_{+}^{1}$ with source $v$. A juxtaposition of oriented edges $w_{1} w_{2}$ is said to be admissible if $w_{2} \neq \iota\left(w_{1}\right)$ and $r\left(w_{1}\right)=s\left(w_{2}\right)$. A (finite, infinite, doubly infinite) walk in a directed graph $E$ is an admissible (finite, infinite, doubly infinite) sequence of elements in $E^{1}$.

We denote by $\mathcal{W}^{n}(E)$ the set of walks of length $n$, by $\mathcal{W}^{*}(E)=\cup_{n} \mathcal{W}^{n}(E)$, by $\mathcal{W}^{+}(E)$ the set of infinite walks, and by $\mathcal{W}(E)$ the set of doubly infinite walks. A directed graph is a directed tree if, for any two vertices, there exists a unique walk in $\mathcal{W}^{*}(E)$ connecting them.

The edge matrix $A_{+}$of a locally finite (or row finite) directed graph is an $\left(\# E_{+}^{1}\right) \times\left(\# E_{+}^{1}\right)$ (possibly infinite) matrix. The entries $A_{+}\left(w_{i}, w_{j}\right)$ satisfy $A_{+}\left(w_{i}, w_{j}\right)=1$ if $w_{i} w_{j}$ is an admissible path, and $A_{+}\left(w_{i}, w_{j}\right)=0$ otherwise. The directed edge matrix of $E$ is a $\# E^{1} \times \# E^{1}$ (possibly infinite) matrix with entries $A\left(w_{i}, w_{j}\right)=1$ if $w_{i} w_{j}$ is an admissible walk and $A\left(w_{i}, w_{j}\right)=0$ otherwise.

Even when not explicitly stated, all Hilbert spaces and algebras of operators we consider will be separable, i.e. they admit a dense (in the norm topology) countable subset. 


\section{A dynamical theory at infinity.}

Let $\Gamma \subset \operatorname{PSL}(2, \mathbb{C})$ be a Schottky group of rank $g \geq 2$. Given a choice of a set of generators $\left\{g_{i}\right\}_{i=1}^{g}$ for $\Gamma$, there is a bijection between the elements of $\Gamma$ and the set of all admissible walks in the Cayley graph of $\Gamma$, namely reduced words in the $\left\{g_{i}\right\}_{i=1}^{2 g}$, where we use the notation $g_{i+g}:=g_{i}^{-1}$, for $i=1, \ldots, g$.

In the following we consider the sets $\mathcal{S}^{+}$and $\mathcal{S}$ of resp. right-infinite, doubly infinite admissible sequences in the $\left\{g_{i}\right\}_{i=1}^{2 g}$ :

$$
\begin{gathered}
\mathcal{S}^{+}=\left\{a_{0} a_{1} \ldots a_{\ell} \ldots \mid a_{i} \in\left\{g_{i}\right\}_{i=1}^{2 g}, a_{i+1} \neq a_{i}^{-1}, \forall i \in \mathbb{N}\right\}, \\
\mathcal{S}=\left\{\ldots a_{-m} \ldots a_{-1} a_{0} a_{1} \ldots a_{\ell} \ldots \mid a_{i} \in\left\{g_{i}\right\}_{i=1}^{2 g}, a_{i+1} \neq a_{i}^{-1}, \forall i \in \mathbb{Z}\right\} .
\end{gathered}
$$

The admissibility condition simply means that we only allow "reduced" words in the generators, without cancellations.

On the space $\mathcal{S}$ we consider the topology generated by the sets $W^{s}(x, \ell)=\left\{y \in \mathcal{S} \mid x_{k}=y_{k}, k \geq \ell\right\}$, and $W^{u}(x, \ell)=\left\{y \in \mathcal{S} \mid x_{k}=y_{k}, k \leq \ell\right\}$ for $x \in \mathcal{S}$ and $\ell \in \mathbb{Z}$.

There is a two-sided shift operator $T$ acting on $\mathcal{S}$ as the map

$$
\begin{aligned}
& \left.\begin{array}{llllllllll}
T( & \ldots & a_{-m} & \ldots & a_{-1} & a_{0} & a_{1} & \ldots & a_{\ell} & \ldots
\end{array}\right)=
\end{aligned}
$$

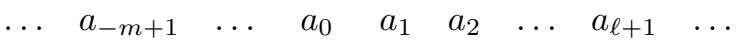

Definition 3.1 A subshift of finite type $\left(\mathcal{S}_{A}, T\right)$ consists of all doubly infinite sequences in the elements of a given finite set $W$ (alphabet) with the admissibility condition specified by a $\# W \times \# W$ elementary matrix,

$$
\mathcal{S}_{A}=\left\{\left(w_{k}\right)_{k \in \mathbb{Z}}: w_{k} \in W, A\left(w_{k}, w_{k+1}\right)=1\right\},
$$

and with the action of the invertible shift $(T w)_{k}=w_{k+1}$.

Lemma 3.2 The space $\mathcal{S}$ can be identified with the subshift of finite type $\mathcal{S}_{A}$ with the symmetric $2 g \times 2 g$ matrix A given by the directed edge matrix of the Cayley graph of $\Gamma$.

The two-sided shift operator $T$ on $\mathcal{S}$ of 3.3 decomposes $\mathcal{S}$ in a product of expanding and contracting directions, so that $(\mathcal{S}, T)$ is a Smale space.

The following topological space is defined in terms of the Smale space $(\mathcal{S}, T)$ and will be considered as a geometric realization of the "dual graph" associated to the fiber at arithmetic infinity of the arithmetic surface $\mathcal{X}$.

Definition 3.3 The mapping torus (suspension flow) of the dynamical system $(\mathcal{S}, T)$ is defined as

$$
\mathcal{S}_{T}:=\mathcal{S} \times[0,1] /(x, 0) \sim(T x, 1)
$$

The space $\mathcal{S}_{T}$ is a very natural space associated to the noncommutative space

$$
\Lambda_{\Gamma} \times_{\Gamma} \Lambda_{\Gamma} \simeq \mathcal{S} / \mathbb{Z}
$$

with $\mathbb{Z}$ acting via the invertible shift $T$ of (3.3), namely the homotopy quotient (cf. [3] [6] $)$,

$$
\mathcal{S}_{T}=\mathcal{S} \times_{\mathbb{Z}} \mathbb{R}
$$

Namely, it is a commutative space that provides, up to homotopy, a geometric model for (3.5), where the noncommutative space (3.5) can be identified with the quotient space of a foliation (3.6) whose generic leaf is contractible (a copy of $\mathbb{R}$ ). 


\section{1 (Co)homology of $\mathcal{S}_{T}$.}

In this paragraph we give an explicit description of the (co)homology $H^{1}\left(\mathcal{S}_{T}, \mathbb{Z}\right)$.

The shift $T$ acting on $\mathcal{S}$ induces an automorphism of the $C^{*}$-algebra of continuous functions $C(\mathcal{S})$. With an abuse of notation we still denote it by $T$. Consider the crossed product $C^{*}$-algebra $C(\mathcal{S}) \rtimes_{T} \mathbb{Z}$. This is a suitable norm completion of $C(\mathcal{S})\left[T, T^{-1}\right]$ with product $(V * W)_{k}=\sum_{r \in \mathbb{Z}} V_{k} \cdot\left(T^{r} W_{r+k}\right)$, for $V=\sum_{k} V_{k} T^{k}, W=\sum_{k} W_{k} T^{k}$, and $V * W=\sum_{k}(V * W)_{k} T^{k}$. The $K$-theory group $K_{0}\left(\mathrm{C}(\mathcal{S}) \rtimes_{T} \mathbb{Z}\right)$ is described by the co-invariants of the action of $T$ (cf. 4, [27]).

Theorem 3.4 The cohomology $H^{1}\left(\mathcal{S}_{T}\right)$ satisfies the following properties

1. There is an identification of $H^{1}\left(\mathcal{S}_{T}, \mathbb{Z}\right)$ with the $K_{0}$-group of the crossed product $\mathrm{C}^{*}$-algebra for the action of $T$ on $\mathcal{S}$,

$$
H^{1}\left(\mathcal{S}_{T}, \mathbb{Z}\right) \cong K_{0}\left(\mathrm{C}(\mathcal{S}) \rtimes_{T} \mathbb{Z}\right) .
$$

2. The identification (3.7) endows $H^{1}\left(\mathcal{S}_{T}, \mathbb{Z}\right)$ with a filtration by free abelian groups $F_{0} \hookrightarrow F_{1} \hookrightarrow$ $\cdots F_{n} \hookrightarrow \cdots$, with $\operatorname{rank} F_{0}=2 g$ and $\operatorname{rank} F_{n}=2 g(2 g-1)^{n-1}(2 g-2)+1$, for $n \geq 1$, so that

$$
H^{1}\left(\mathcal{S}_{T}, \mathbb{Z}\right)=\underset{n}{\lim } F_{n}
$$

3. This filtration is induced by a filtration $\mathcal{P}_{n}$ on the locally constant functions $\mathrm{C}\left(\mathcal{S}^{+}, \mathbb{Z}\right)$ which depend on "future coordinates", with $\mathcal{P}_{n}$ given by locally constant functions that depend only on the first $n+1$ coordinates. The Pimsner-Voiculescu exact sequence for the K-theory (3.7),

$$
0 \rightarrow \mathbb{Z} \rightarrow \mathrm{C}(\mathcal{S}, \mathbb{Z}) \stackrel{\delta=1-T}{\longrightarrow} \mathrm{C}(\mathcal{S}, \mathbb{Z}) \rightarrow H^{1}\left(\mathcal{S}_{T}, \mathbb{Z}\right) \rightarrow 0 .
$$

4. The cohomology $H^{1}\left(\mathcal{S}_{T}, \kappa\right)=H^{1}\left(\mathcal{S}_{T}, \mathbb{Z}\right) \otimes \kappa$, for $\kappa=\mathbb{R}$ or $\mathbb{C}$, is computed by

$$
0 \rightarrow \mathbb{C} \rightarrow \mathcal{P} \otimes \kappa \stackrel{\delta}{\longrightarrow} \mathcal{P} \otimes \kappa \rightarrow H^{1}\left(\mathcal{S}_{T}, \mathbb{C}\right) \rightarrow 0 .
$$

The vector space $\mathcal{P} \otimes \kappa$, for $\mathcal{P}=\mathrm{C}\left(\mathcal{S}^{+}, \mathbb{Z}\right)=\varliminf_{n} \mathcal{P}_{n}$, admits a Hilbert space completion $\mathcal{L}=$ $L^{2}\left(\Lambda_{\Gamma}, \mu\right)$, where $\mu$ is the Patterson-Sullivan measure on the limit set $\Lambda_{\Gamma}$ (cf. [31]) satisfying

$$
\left(\gamma^{*} d \mu\right)(x)=\left|\gamma^{\prime}(x)\right|^{\delta_{H}} d \mu(x), \quad \forall \gamma \in \Gamma .
$$

Notice that the adjoint $\delta^{*}$ in the $L^{2}$-inner product of the coboundary $\delta$ of the cohomology $H^{1}\left(\mathcal{S}_{T}\right)$ is an important operator associated to the dynamics of the (one sided) shift $T$ on the limit set $\Lambda_{\Gamma}$, namely, the operator $1-\mathcal{R}$, where $\mathcal{R}$ is the Perron-Frobenius operator of $T$. This is the analog of the Gauss-Kuzmin operator studied in [24] 25] in the case of modular curves.

For simplicity of notation, in the following we will use the same notation $\mathcal{P}$ and $\mathcal{P}_{n}$ for the $\mathbb{Z}$ modules and for the $\kappa$-vector spaces. It will be clear from the context which one we refer to. We identify the vector spaces $\mathcal{P}_{n}$ with finite dimensional subspaces of $L^{2}\left(\Lambda_{\Gamma}, \mu\right)$, by identifying locally constant functions on $\mathcal{S}^{+}$with locally constant functions on $\Lambda_{\Gamma}$.

The following result computes the first homology of $\mathcal{S}_{T}$.

Proposition 3.5 The homology group $H_{1}\left(\mathcal{S}_{T}, \mathbb{Z}\right)$ has a filtration by free abelian groups $\mathcal{K}_{N}$,

$$
H_{1}\left(\mathcal{S}_{T}, \mathbb{Z}\right)=\underset{N}{\lim } \mathcal{K}_{N}
$$

with

$$
K_{N}=\operatorname{rank}\left(\mathcal{K}_{N}\right)=\left\{\begin{array}{lr}
(2 g-1)^{N}+1 & N \text { even } \\
(2 g-1)^{N}+(2 g-1) & N \text { odd }
\end{array}\right.
$$


The group $H_{1}\left(\mathcal{S}_{T}, \mathbb{Z}\right)$ can also be written as

$$
H_{1}\left(\mathcal{S}_{T}, \mathbb{Z}\right)=\oplus_{N=0}^{\infty} \mathcal{R}_{N}
$$

where $\mathcal{R}_{n}$ is a free abelian group of ranks $R_{1}=2 g$ and

$$
R_{N}=\operatorname{rank}\left(\mathcal{R}_{N}\right)=\frac{1}{N} \sum_{d \mid N} \mu(d)(2 g-1)^{N / d},
$$

for $N>1$, with $\mu$ the Möbius function. This is isomorphic to a free abelian group on countably many generators.

Thus, the $\mathbb{Z}$-modules $F_{n}$ are obtained as quotients $\mathcal{P}_{n} / \delta \mathcal{P}_{n-1}$, for $\delta(f)=f-f \circ T$, where $\mathcal{P}=\cup_{n} \mathcal{P}_{n}$ is the module of continuous $\mathbb{Z}$-valued functions on $\mathcal{S}$ that depend only on "future coordinates". The $\mathbb{Z}$ modules $\mathcal{K}_{N}$ are generated by all admissible words $a_{0} \ldots a_{N}$ such that the word $a_{N} a_{0}$ is also admissible. Combining Theorem 3.4 with Proposition 3.5] we can compute explicitly the pairing of homology and cohomology for $\mathcal{S}_{T}$.

Proposition 3.6 Let $F_{n}$ and $\mathcal{K}_{N}$ be the filtrations defined, respectively, in Theorem 3.4 and Proposition 3.5. There is a pairing

$$
\langle\cdot, \cdot\rangle: F_{n} \times \mathcal{K}_{N} \rightarrow \mathbb{Z} \quad\langle[f], x\rangle=N \cdot f(\bar{x}),
$$

with $x=a_{0} \ldots a_{N}$. Here the representative $f \in[f]$ is a function that depends on the first $n+1$ terms $a_{0} \ldots a_{n}$ of sequences in $\mathcal{S}$, and $\bar{x}$ is the truncation of the periodic sequence $\overline{a_{0} \ldots a_{N}}$ after the first $n$ terms. This pairing descends to the direct limits of the filtrations, where it agrees with the classical cohomology/homology pairing

$$
\langle\cdot, \cdot\rangle: H^{1}\left(\mathcal{S}_{T}, \mathbb{Z}\right) \times H_{1}\left(\mathcal{S}_{T}, \mathbb{Z}\right) \rightarrow \mathbb{Z}
$$

\subsection{Dynamical (co)homology.}

We define the dynamical cohomology $H_{d y n}^{1}$ as the graded vector space given by the sum of the graded pieces of the filtration of $H^{1}\left(\mathcal{S}_{T}\right)$, introduced in Theorem 3.4 These graded pieces $\mathrm{Gr}_{n}$ are considered with coefficients in the $n$-th Hodge-Tate twist $\mathbb{R}(n)$, for $n \in \mathbb{Z}$. Similarly, we define the dynamical homology $H_{1}^{d y n}$ as the graded vector space given by the sum of the terms in the filtration of $H_{1}\left(\mathcal{S}_{T}\right)$, introduced in Proposition 3.5. These vector spaces are again considered with twisted $\mathbb{R}(n)$-coefficients. The pair

$$
H_{d y n}^{1} \oplus H_{1}^{d y n}
$$

provides a geometric setting, defined in terms of the dynamics of the shift operator $T$, which contains a copy of the Archimedean cohomology of [10] and of its dual.

Definition 3.7 Let $H^{1}\left(\mathcal{S}_{T}, \kappa\right)=\lim _{n} F_{n}$, for a filtration $F_{n}$ as in Theorem 3.4 with real or complex coefficients. Let $\mathrm{Gr}_{n}=F_{n} / F_{n-1}$ be the corresponding graded pieces, with $\mathrm{Gr}_{0}=F_{0}$.

1. We define a graded linear subspace $\mathcal{V}$ of the Hilbert space $\mathcal{L}$, as the span of the elements

$$
\hat{\Pi}_{n} \chi_{\mathcal{S}^{+}\left(w_{n, k}\right)},
$$

with $\chi_{\mathcal{S}^{+}\left(w_{n, k}\right)}$ the characteristic function of $\mathcal{S}^{+}\left(w_{n, k}\right) \subset \mathcal{S}^{+}$with $w_{n, k}:=a_{0} a_{1} \ldots a_{n-1}=$ $\underbrace{g_{k} g_{k} \ldots g_{k}}_{n-\text { times }}$. The operator $\hat{\Pi}_{n}$ is the projection $\hat{\Pi}_{n}=\Pi_{n}-\Pi_{n-1}$, with $\Pi_{n}$ the orthogonal projection of $\mathcal{L}$ onto $\mathcal{P}_{n}$. 
2. We define the dynamical cohomology as

$$
H_{d y n}^{1}:=\oplus_{n \leq 0} g r_{2 n}^{\Gamma} H_{d y n}^{1}
$$

where we set

$$
g r_{2 n}^{\Gamma} H_{d y n}^{1}:=\operatorname{Gr}_{-n} \otimes_{\mathbb{R}} \mathbb{R}(n)
$$

with $\mathbb{R}(n)=(2 \pi \sqrt{-1})^{n} \mathbb{R}$.

Furthermore, we define the graded subspace of $H_{d y n}^{1}$

$$
\overline{\mathcal{V}}:=\oplus_{n \leq 0} g r_{2 n}^{\Gamma} \overline{\mathcal{V}}
$$

where $\operatorname{gr}{ }_{2 n}^{\Gamma} \overline{\mathcal{V}}$ is generated by the elements $(2 \pi \sqrt{-1})^{n} \chi_{-n+1, k}$, for $\chi_{n, k}:=\left[\chi_{\mathcal{S}^{+}\left(w_{n, k}\right)}\right] \in \mathrm{Gr}_{n-1}$.

3. The dynamical homology $H_{1}^{\text {dyn }}$ is defined as

$$
H_{1}^{d y n}:=\oplus_{n \geq 1} g r_{2 n}^{\Gamma} H_{1}^{d y n},
$$

where we set

$$
g r_{2 n}^{\Gamma} H_{1}^{d y n}:=\mathcal{K}_{n-1} \otimes_{\mathbb{R}} \mathbb{R}(n) .
$$

We also define $\mathcal{W} \subset H_{1}^{d y n}$ as the graded sub-space $\mathcal{W}=\oplus_{n \geq 1} g r_{2 n}^{\Gamma} \mathcal{W}$, where $\operatorname{gr}_{2 n}^{\Gamma} \mathcal{W}$ is generated by the $2 g$ elements $(2 \pi \sqrt{-1})^{n} \underbrace{g_{k} g_{k} \ldots g_{k}}_{n-\text { times }}$.

The choice of indexing the grading by $g r_{2 n}^{\Gamma}$ instead of $g r_{n}^{\Gamma}$ is motivated by comparison to the grading on the cohomological construction of [10].

In [11, we showed that the subspaces $\mathcal{V}$ and $\overline{\mathcal{V}}$ realize copies of the Archimedean cohomology of [10] embedded in the space of cochains $\mathcal{L}$ of the dynamical cohomology and in the dynamical cohomology itself, while the pair $\overline{\mathcal{V}} \oplus \mathcal{W}$ realize a copy of the cohomology of the cone of the "local monodromy map" $N$ of [10] inside the pair of dynamical cohomology and homology $H_{d y n}^{1} \oplus H_{1}^{d y n}$. The isomorphism between $\mathcal{V}$ and the Archimedean cohomology is realized by a natural choice of a basis of holomorphic differentials for the Archimedean cohomology, constructed from the data of the Schottky uniformozation as in 22.

An explicit geometric description for the space $\mathcal{V}$ in terms of geodesics in $\mathfrak{X}_{\Gamma}$ is obtained by interpreting the characteristic function $\chi_{\mathcal{S}^{+}\left(w_{n, k}\right)}$ as the "best approximation" within $\mathcal{P}_{n}$ to a distribution supported on the periodic sequence of period $g_{k}$,

$$
f\left(g_{k} g_{k} g_{k} g_{k} g_{k} \ldots\right)=1 \text { and } f\left(a_{0} a_{1} a_{2} a_{3} \ldots\right)=0 \text { otherwise. }
$$

Such periodic sequence $g_{k} g_{k} g_{k} g_{k} g_{k} \ldots$ describes the closed geodesic in $\mathfrak{X}_{\Gamma}$ that is the oriented core of one of the handles in the handlebody. Thus, the subspace $g r_{2 n}^{\Gamma} \mathcal{V} \subset \mathcal{P}_{n}$ is spanned by the "best approximations" within $\mathcal{P}_{n}$ to cohomology classes supported on the core handles of the handlebody. In other words, this interpretation views the index $n \leq 0$ of the graded structure $\mathcal{V}=\oplus_{n} g r_{2 n}^{\Gamma} \mathcal{V}$ as a measure of "zooming in", with increasing precision for larger $|n|$, on the core handles of the handlebody $\mathfrak{X}_{\Gamma}$.

\subsection{A spectral triple from dynamics.}

Recall that a spectral triple consists of the following data (cf. [9]).

Definition 3.8 a spectral triple $(\mathcal{A}, \mathcal{H}, D)$ consists of a $\mathrm{C}^{*}$-algebra $\mathcal{A}$ with a representation

$$
\rho: \mathcal{A} \rightarrow \mathcal{B}(\mathcal{H})
$$

as bounded operators on a Hilbert space $\mathcal{H}$, and an operator $D$ (called the Dirac operator) on $\mathcal{H}$, which satisfies the following properties: 


\section{1. $D$ is self-adjoint.}

2. For all $\lambda \notin \mathbb{R}$, the resolvent $(D-\lambda)^{-1}$ is a compact operator on $\mathcal{H}$.

3. The commutator $[D, a]$ is a bounded operator on $\mathcal{H}$, for all $a \in \mathcal{A}_{0}$, a dense involutive subalgebra of $\mathcal{A}$.

We consider the Cuntz-Krieger algebra $\mathcal{O}_{A}\left(c f\right.$. [13] [14]) defined as the universal $\mathrm{C}^{*}$-algebra generated by partial isometries $S_{1}, \ldots, S_{2 g}$, satisfying the relations

$$
\begin{gathered}
\sum_{j} S_{j} S_{j}^{*}=I \\
S_{i}^{*} S_{i}=\sum_{j} A_{i j} S_{j} S_{j}^{*},
\end{gathered}
$$

where $A=\left(A_{i j}\right)$ is the $2 g \times 2 g$ transition matrix of the subshift of finite type $(\mathcal{S}, T)$, namely the matrix whose entries are $A_{i j}=1$ whenever $|i-j| \neq g$, and $A_{i j}=0$ otherwise.

The algebra $\mathcal{O}_{A}$ can be also described in terms of the action of the free group $\Gamma$ on its limit set $\Lambda_{\Gamma}$ (cf. 28], 30]), so that we can regard $\mathcal{O}_{A}$ as a noncommutative space replacing the classical quotient $\Lambda_{\Gamma} / \Gamma$. In fact, the action of $\Gamma$ on $\Lambda_{\Gamma} \subset \mathbb{P}^{1}(\mathbb{C})$ determines a unitary representation of $\mathcal{O}_{A}$ on the Hilbert space $L^{2}\left(\Lambda_{\Gamma}, \mu\right)$, given by

$$
\left(T_{\gamma^{-1}} f\right)(x):=\left|\gamma^{\prime}(x)\right|^{\delta_{H} / 2} f(\gamma x), \quad \text { and } \quad\left(P_{\gamma} f\right)(x):=\chi_{\gamma}(x) f(x),
$$

where $\delta_{H}$ is the Hausdorff dimension of $\Lambda_{\Gamma}$ and the element $\gamma \in \Gamma$ is identified with a reduced word in the generators $\left\{g_{j}\right\}_{j=1}^{g}$ and their inverses, and $\chi_{\gamma}$ is the characteristic function of the cylinder $\Lambda_{\Gamma}(\gamma)$ of all (right) infinite reduced words that begin with the word $\gamma$. This determines an identification of $\mathcal{O}_{A}$ with the (reduced) crossed product $\mathrm{C}^{*}$-algebra, $\mathcal{O}_{A} \cong C\left(\Lambda_{\Gamma}\right) \rtimes \Gamma$.

We then consider on the Hilbert space $\mathcal{L}=L^{2}\left(\Lambda_{\Gamma}, \mu\right)$, the unbounded linear self adjoint operator $D: \mathcal{L} \rightarrow \mathcal{L}$ given by the grading operator of the filtration $\mathcal{P}_{n}$, namely,

$$
D=\sum_{n} n \hat{\Pi}_{n}
$$

The restriction of this operator to the subspace $\mathcal{V}$ of the dynamical cohomology, isomorphic to the Archimedean cohomology of [10], agrees with the "Frobenius" operator $\Phi$ considered in [10], which computes the local factor as a regularized determinant as in [15.

We extend the operator (3.22) to an operator $\mathcal{D}$ on $\mathcal{H}=\mathcal{L} \oplus \mathcal{L}$ as

$$
\left.\mathcal{D}\right|_{\mathcal{L} \oplus 0}=\left.\sum_{n}(n+1)\left(\hat{\Pi}_{n} \oplus 0\right) \quad \mathcal{D}\right|_{0 \oplus \mathcal{L}}=-\sum_{n} n\left(0 \oplus \hat{\Pi}_{n}\right) .
$$

The presence of a shift by one in the grading operator, reflects the shift by one in the grading that appears in the duality isomorphisms on the cohomology of the cone of the monodromy map $N$ as in Proposition 4.8 of [10]. The Dirac operator (3.23) takes into account the presence of this shift.

There is another possible natural choice for the sign of the Dirac operator, instead of the one in (3.23). Instead of being determined by the sign of the operator $\Phi$ on the archimedean cohomology and its dual, this other choice is determined by the duality map that exists on the complex of [10], realized by powers of the monodromy map ( $c f$. [10, Proposition 4.8). In this case, the sign would then be given by the operator

$$
F=\left(\begin{array}{cc}
0 & 1 \\
1 & 0
\end{array}\right)
$$

that exchanges the two copies of $\mathcal{L}$ in $\mathcal{H}$.

We assume that the Schottky group $\Gamma$ has limit set $\Lambda_{\Gamma}$ of Hausdorff dimension $\delta_{H}<1$. Then the data above define the "dynamical spectral triple" at arithmetic infinity. 
Theorem 3.9 The data $\left(\mathcal{O}_{A}, \mathcal{H}, \mathcal{D}\right)$, where the algebra $\mathcal{O}_{A}$ acts diagonally on $\mathcal{H}=\mathcal{L} \oplus \mathcal{L}$, and the operator $\mathcal{D}$ is given by (3.23) form a spectral triple in the sense of Connes, as in Definition 3.8.

The bound on the commutators with the generators $S_{i}$ of $\mathcal{O}_{A}$ and their adjoints, is obtained in [11] in terms of the Poincaré series of the Schottky group.

The spectral triple defined this way appears to be related to spectral triples for AF algebras, recently introduced in 1 1 . In such constructions the Dirac operator generalizes the grading operator (3.22), by operators of the form $D=\sum \alpha_{n} \hat{\Pi}_{n}$, where $\hat{\Pi}_{n}=\Pi_{n}-\Pi_{n-1}$ are the projections associated to a filtration of the AF algebra, and the coefficients $\alpha_{n}$ given by a sequence of positive real numbers, satisfying certain growth conditions.

The $C^{*}$-algebra $\mathrm{C}\left(\Lambda_{\Gamma}\right)$ is a commutative $\mathrm{AF}$-algebra (approximately finite dimensional), obtained as the direct limit of the finite dimensional commutative $C^{*}$-algebras generated by characteristic functions of a covering of $\Lambda_{\Gamma}$. This gives rise to the filtration $\mathcal{P}_{n}$ in Theorem 3.4 hence our choice of Dirac operator fits into the setting of [1] for the AF algebra $\mathrm{C}\left(\Lambda_{\Gamma}\right)$. On the other hand, while in the construction of [1] the eigenvalues $\alpha_{n}$ can be chosen sufficiently large, so that the resulting spectral triple for the AF algebra would be finitely summable, when we consider the Cuntz-Krieger algebra $\mathcal{O}_{A} \cong \mathrm{C}\left(\Lambda_{\Gamma}\right) \rtimes \Gamma$, the boundedness of commutators (condition 3. of Definition 3.8) can only be satisfied for the special choice of $\alpha_{n}=c n$, with $c$ a constant, $c f$. Remark 2.2 of [1, which does not yield a finitely summable spectral triple. The reason for this lies in a well known result of Connes 7 which shows that non amenable discrete groups (as is the case for the Schottky group $\Gamma$ ) do not admit finitely summable spectral triples. Thus, if the dense subalgebra of $\mathcal{O}_{A}$ with which $\mathcal{D}$ has bounded commutators contains group elements, then the Dirac operator $\mathcal{D}$ cannot be finitely summable.

In our construction, the choice of $\operatorname{sign}$ for $\mathcal{D}$ is prescribed by the graded structure of the cohomology theory of [10] and by the identification of the Archimedean cohomology of [10] and its dual with subspaces of the dynamical cohomology and homology as in 11]. This way, the requirement that the Dirac operator agrees with the operator $\Phi$ of [10] on these subspaces fixes the choice of the sign of the Dirac operator, which carries the topological information on the noncommutative manifold. In the construction of spectral triples for AF algebras of [1] only the metric aspect of the spectral triple is retained, that is, the operator considered is of the form $|D|$, while the sign is not discussed.

A possible way to refine the construction of the spectral triple and deal with the lack of finite summability is through the fact that the Cuntz-Krieger algebra $\mathcal{O}_{A}$ has a second description as a crossed product algebra. Namely, up to stabilization (i.e. tensoring with compact operators) we have

$$
\mathcal{O}_{A} \simeq \mathcal{F}_{A} \rtimes_{T} \mathbb{Z}
$$

where $\mathcal{F}_{A}$ is an approximately finite dimensional (AF) algebra, $c f$. 13], 14. This algebra can be described in terms of a groupoid $\mathrm{C}^{*}$-algebra associated to the "unstable manifold" in the Smale space $(\mathcal{S}, T)$. In fact, consider the algebra $\mathcal{O}_{A}^{a l g}$ generated algebraically by the $S_{i}$ and $S_{i}^{*}$ subject to the Cuntz-Krieger relations (3.19) (3.20). Elements in $\mathcal{O}_{A}^{\text {alg }}$ are linear combinations of monomials $S_{\mu} S_{\nu}^{*}$, for multi-indices $\mu, \nu, c f$. [14]. The AF algebra $\mathcal{F}_{A}$ is generated by elements $S_{\mu} S_{\nu}^{*}$ with $|\mu|=|\nu|$, and is filtered by finite dimensional algebras $\mathcal{F}_{A, n}$ generated by elements of the form $S_{\mu} P_{i} S_{\nu}^{*}$ with $|\mu|=|\nu|=n$ and $P_{i}=S_{i} S_{i}^{*}$ the range projections, and embeddings determined by the matrix $A$. The commutative algebra $\mathrm{C}\left(\Lambda_{\Gamma}\right)$ sits as a subalgebra of $\mathcal{F}_{A}$ generated by all range projections $S_{\mu} S_{\mu}^{*}$. The embedding is compatible with the filtration and with the action of the shift $T$, which is implemented on $\mathcal{F}_{A}$ by the transformation $a \mapsto \sum_{i} S_{i} a S_{i}^{*}$. (cf. 14.)

The fact that the algebra can be written in the form (3.24) implies that, by Connes' result on hyperfiniteness 7, it may carry a finitely summable spectral triple. It is an interesting problem whether the construction of a finitely summable triple can be carried out in a way that is of arithmetic significance.

\subsection{Local factor}

For an arithmetic variety $\mathcal{X}$ over Spec $\mathbb{Z}$, the "Archimedean factor" (local factor at arithmetic infinity) $L_{\kappa}\left(H^{m}, s\right)$ is a product of Gamma functions, with exponents and arguments that depend on the Hodge 
structure $H^{m}=H^{m}(X, \mathbb{C})=\oplus_{p+q=m} H^{p, q}$. More precisely, (cf. [29])

$$
L_{\kappa}\left(H^{m}, s\right)= \begin{cases}\prod_{p, q} \Gamma_{\mathbb{C}}(s-\min (p, q))^{h^{p, q}} & \kappa=\mathbb{C} \\ \prod_{p<q} \Gamma_{\mathbb{C}}(s-p)^{h^{p, q}} \prod_{p} \Gamma_{\mathbb{R}}(s-p)^{h^{p+}} \Gamma_{\mathbb{R}}(s-p+1)^{h^{p-}} & \kappa=\mathbb{R},\end{cases}
$$

where the $h^{p, q}$, with $p+q=m$, are the Hodge numbers, $h^{p, \pm}$ is the dimension of the $\pm(-1)^{p}$-eigenspace of de Rham conjugation on $H^{p, p}$, and

$$
\Gamma_{\mathbb{C}}(s):=(2 \pi)^{-s} \Gamma(s) \quad \Gamma_{\mathbb{R}}(s):=2^{-1 / 2} \pi^{-s / 2} \Gamma(s / 2) .
$$

Deninger produced a unified description of the factors at arithmetic infinity and at the finite primes, in the form of a Ray-Singer determinant ( $c f$. [15]). The factor (3.25) satisfies

$$
L_{\kappa}\left(H^{m}, s\right)=\operatorname{det}_{\infty}\left(\frac{1}{2 \pi}(s-\Phi) \mid \mathcal{\nu}^{m}\right)^{-1},
$$

where $\mathcal{V}^{m}$ is an infinite dimensional vector space. The zeta regularized determinant of an unbounded self adjoint operator $T$ is defined as $\operatorname{det}_{\infty}(s-T)=\exp \left(-\left.\frac{d}{d z} \zeta_{T}(s, z)\right|_{z=0}\right)$.

In [10, the spaces $\mathcal{V}$ are identified with inertia invariants of a double complex of real Tate-twisted differential forms on $X_{/ \mathbb{C}}$ with suitable cutoffs. Namely, such complex is endowed with the action of an endomorphism $N$, which represents a "logarithm of the local monodromy at arithmetic infinity", and the spaces $\mathcal{V}$ are identified with the kernel of the map $N$ on the hypercohomology. In particular, in the case of an arithmetic surface, we showed in [1] that the Archimedean cohomology group $\mathcal{V}^{1}$ is identified with a subspace $\mathcal{V}$ of $\mathcal{P}$ and of the dynamical cohomology $H_{d y n}^{1}$.

The dynamical spectral triple of Theorem 3.9 is not finitely summable. However, it is possible to recover from these data the local factor at arithmetic infinity (3.26) for $m=1$.

Proposition 3.10 Consider the zeta functions

$$
\zeta_{\pi(\mathcal{V}), D}(s, z):=\sum_{\lambda \in \operatorname{Spec}(D)} \operatorname{Tr}(\pi(\mathcal{V}) \Pi(\lambda, D))(s-\lambda)^{-z},
$$

for $\pi(\mathcal{V})$ the orthogonal projection on the norm closure of $\mathcal{V}$ in $\mathcal{L}$, and

$$
\zeta_{\pi\left(\mathcal{V}, \bar{F}_{\infty}=i d\right), D}(s, z):=\sum_{\lambda \in \operatorname{Spec}(D)} \operatorname{Tr}\left(\pi\left(\mathcal{V}, \bar{F}_{\infty}=i d\right) \Pi(\lambda, D)\right)(s-\lambda)^{-z},
$$

for $\pi\left(\mathcal{V}, \bar{F}_{\infty}=i d\right)$ the orthogonal projection on the norm closure of $\mathcal{V}^{\bar{F}_{\infty}=i d}$. The corresponding regularized determinants satisfy

$$
\begin{gathered}
\exp \left(-\left.\frac{d}{d z} \zeta_{\pi(\mathcal{V}), D / 2 \pi}(s / 2 \pi, z)\right|_{z=0}\right)^{-1}=L_{\mathbb{C}}\left(H^{1}(X), s\right), \\
\exp \left(-\left.\frac{d}{d z} \zeta_{\pi\left(\mathcal{V}, \bar{F}_{\infty}=i d\right), D / 2 \pi}(s / 2 \pi, z)\right|_{z=0}\right)^{-1}=L_{\mathbb{R}}\left(H^{1}(X), s\right) .
\end{gathered}
$$

Moreover, the operator $\pi(\mathcal{V})$ acts on the range of the spectral projections $\Pi(\lambda, D)$ as certain elements of the algebra $\mathcal{O}_{A}$.

Here $\bar{F}_{\infty}$ is the involution induced by the real structure on $X_{/ \mathbb{R}}$, which corresponds to the change of orientation on the geodesics in $\mathfrak{X}_{\Gamma}$ and on $\mathcal{S}_{T}$. 

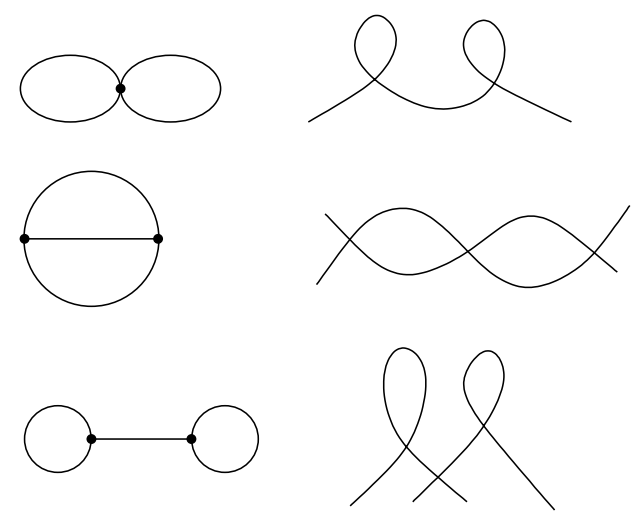

Figure 1: The graphs $\Delta_{\Gamma} / \Gamma$ for genus $g=2$, and the corresponding fibers.

\section{A Dynamical theory for Mumford curves.}

Throughtout this chapter $K$ will denote a finite extension of $\mathbb{Q}_{p}$ and $\Delta_{K}$ the Bruhat-Tits tree associated to $G=\operatorname{PGL}(2, K)$. In the following we recall few results about the action of a Schottky group on a Bruhat-Tits tree and on $\mathrm{C}^{*}$-algebras of graphs. Detailed explanations are contained in [20], 26] and [2, 18, 19, 30].

Recall that the Bruhat-Tits tree is constructed as follows. One considers the set of free $\mathcal{O}$-modules of rank 2: $M \subset V$. Two such modules are equivalent $M_{1} \sim M_{2}$ if there exists an element $\lambda \in K^{*}$, such that $M_{1}=\lambda M_{2}$. The group $\mathrm{GL}(\mathrm{V})$ of linear automorphisms of $V$ operates on the set of such modules on the left: $g M=\{g m \mid m \in M\}, g \in \mathrm{GL}(\mathrm{V})$. Notice that the relation $M_{1} \sim M_{2}$ is equivalent to the condition that $M_{1}$ and $M_{2}$ belong to the same orbit of the center $K^{*} \subset \mathrm{GL}(\mathrm{V})$. Hence, the group $G=\mathrm{GL}(\mathrm{V}) / \mathrm{K}^{*}$ operates (on the left) on the set of classes of equivalent modules.

We denote by $\Delta_{K}^{0}$ the set of such classes and by $\{M\}$ the class of the module $M$. Because $\mathcal{O}$ is a principal ideals domain and every module $M$ has two generators, it follows that

$$
\left\{M_{1}\right\},\left\{M_{2}\right\} \in \Delta_{K}^{0}, M_{1} \supset M_{2} \quad \Rightarrow \quad M_{1} / M_{2} \simeq \mathcal{O} / \mathfrak{m}^{l} \oplus \mathcal{O} / \mathfrak{m}^{k}, \quad l, k \in \mathbb{N} .
$$

The multiplication of $M_{1}$ and $M_{2}$ by elements of $K$ preserves the inclusion $M_{1} \supset M_{2}$, hence the natural number

$$
d\left(\left\{M_{1}\right\},\left\{M_{2}\right\}\right)=|l-k|
$$

is well defined.

The graph $\Delta_{K}$ of the group PGL $(2, K)$ is the infinite graph with set of vertices $\Delta_{K}^{0}$, in which two vertices $\left\{M_{1}\right\},\left\{M_{2}\right\}$ are adjacent and hence connected by an edge if and only if $d\left(\left\{M_{1}\right\},\left\{M_{2}\right\}\right)=1$. (cf. [20] and [26].)

For a Schottky group $\Gamma \subset \operatorname{PGL}(2, K)$ there is a smallest subtree $\Delta_{\Gamma}^{\prime} \subset \Delta_{K}$ containing the axes of all elements of $\Gamma$. The set of ends of $\Delta_{\Gamma}^{\prime}$ in $\mathbb{P}^{1}(K)$ is $\Lambda_{\Gamma}$, the limit set of $\Gamma$. The group $\Gamma$ carries $\Delta_{\Gamma}^{\prime}$ into itself so that the quotient $\Delta_{\Gamma}^{\prime} / \Gamma$ is a finite graph that coincides with the dual graph of the closed fibre of the minimal smooth model of the algebraic curve $C / K$ holomorphically isomorphic to $X_{\Gamma}:=\Omega_{\Gamma} / \Gamma$ (cf. 26] p. 163). There is a smallest tree $\Delta_{\Gamma}$ on which $\Gamma$ acts and such that $\Delta_{\Gamma} / \Gamma$ is the (finite) graph of the specialization of $C$. The curve $C$ is a $k$-split degenerate, stable curve. When the genus of the fibers is at least 2 - i.e. when the Schottky group has at least $g \geq 2$ generators - the curve $X_{\Gamma}$ is called a Schottky-Mumford curve.

The possible graphs $\Delta_{\Gamma} / \Gamma$ and the corresponding fiber for the case of genus 2 are illustrated in Figure 1

To a locally finite directed graph one associates a $\mathrm{C}^{*}$-algebra in the following way. 
A Cuntz-Krieger family consists of a collection $\left\{P_{v}\right\}_{v \in E^{0}}$ of mutually orthogonal projections and a $\left\{S_{w}\right\}_{w \in E_{+}^{1}}$ of partial isometries, satisfying the conditions: $S_{w}^{*} S_{w}=P_{r(w)}$ and, for all $v \in s\left(E_{+}^{1}\right)$, $P_{v}=\sum_{w: s(w)=v} S_{w} S_{w}^{*}$.

The Cuntz-Krieger elements $\left\{P_{v}, S_{w}\right\}$ satisfy the relation $S_{w}^{*} S_{w}=\sum A_{+}(w, \tilde{w}) S_{\tilde{w}} S_{\tilde{w}}^{*}$, with $A_{+}$ the edge matrix of the graph.

One defines a universal $C^{*}$-algebra $C^{*}(E)$ generated by a Cuntz-Krieger family. If $E$ is a finite graph with no sinks, we have $C^{*}(E) \simeq \mathcal{O}_{A_{+}}$, where $\mathcal{O}_{A_{+}}$is the Cuntz-Krieger algebra of the edge matrix $A_{+}$. If the directed graph is a tree $\Delta$, then $C^{*}(\Delta)$ is an AF algebra strongly Morita equivalent to the commutative $C^{*}$-algebra $C_{0}(\partial \Delta)$. A monomorphism of directed trees induces an injective *-homomorphism of the corresponding $C^{*}$-algebras.

If $G \subset \operatorname{Aut}(E)$ is a group acting freely on the directed graph $E$, with quotient graph $E / G$, then the crossed product $C^{*}$-algebra $C^{*}(E) \rtimes G$ is strongly Morita equivalent to $C^{*}(E / G)$. In particular, if $\Delta$ is the universal covering tree of a directed graph $E$ and $\Gamma$ is the fundamental group, then the algebra $C^{*}(E)$ is strongly Morita equivalent to $C_{0}(\partial \Delta) \rtimes \Gamma$.

In the following we consider the Bruhat-Tits tree $\Delta_{K}$ for a fixed finite extension $K$ of $\mathbb{Q}_{p}$, and the corresponding $C^{*}$-algebra $C^{*}\left(\Delta_{K}\right)$, which is strongly Morita equivalent to the abelian $C^{*}$-algebra of complex valued functions $C\left(\mathbb{P}^{1}(K)\right)$.

Notice that the commutative $C^{*}$-algebra $C^{*}\left(X_{K}\right)$ of complex valued continuous functions on the Mumford curve $X_{K}=\Omega_{\Gamma} / \Gamma$ is strongly Morita equivalent to the crossed product $C_{0}\left(\Omega_{\Gamma}\right) \rtimes \Gamma$, with $C_{0}\left(\Omega_{\Gamma}\right)$ a $\Gamma$-invariant ideal of $C\left(\mathbb{P}^{1}(K)\right)$ with quotient algebra $C\left(\Lambda_{\Gamma}\right)$. The algebra $C^{*}\left(\Delta_{\Gamma} / \Gamma\right)$, in turn, is strongly Morita equivalent to $C^{*}\left(\Delta_{\Gamma}\right) \rtimes \Gamma$ and to $C\left(\Lambda_{\Gamma}\right) \rtimes \Gamma$, where $\partial \Delta_{\Gamma}=\Lambda_{\Gamma}$. Similarly, one sees that the algebra $C^{*}\left(\Delta_{K} / \Gamma\right)$ is strongly Morita equivalent to the crossed product algebra $C^{*}\left(\Delta_{K}\right) \rtimes \Gamma$, which in turn is strongly Morita equivalent to $C^{*}\left(\mathbb{P}^{1}(K)\right) \rtimes \Gamma$.

Thus, up to Morita equivalence, the graph algebra $C^{*}\left(\Delta_{K} / \Gamma\right)$ can be regarded as a way of extending the commutative $C^{*}$-algebra $C^{*}\left(X_{K}\right)$ (functions on the Mumford curve) by the Cuntz-Krieger algebra $C^{*}\left(\Delta_{\Gamma} / \Gamma\right)$ associated to the edge matrix of the finite graph $\Delta_{\Gamma} / \Gamma$.

In this paragraph we introduce a dynamical system associated to the space $\mathcal{W}(\Delta / \Gamma)$ of walks on the directed tree $\Delta$ on which $\Gamma$ acts. In particular, we are interested in the cases when $\Delta=\Delta_{K}, \Delta_{\Gamma}$.

For $\Delta=\Delta_{\Gamma}$, we obtain a subshift of finite type associated to the action of the Schottky group $\Gamma$ on the limit set $\Lambda_{\Gamma}$, of the type that was considered in [11.

Let $\bar{V} \subset \Delta_{\Gamma}$ be a finite subtree whose set of edges consists of one representative for each $\Gamma$-class. This is a fundamental domain for $\Gamma$ in the weak sense (following the notation of 20]), since some vertices may be identified under the action of $\Gamma$. Correspondingly, $V \subset \mathbb{P}^{1}(K)$ is the set of ends of all infinite paths starting at points in $\bar{V}$.

Consider the set $\mathcal{W}\left(\Delta_{\Gamma} / \Gamma\right)$ of doubly infinite walks on the finite graph $\Delta_{\Gamma} / \Gamma$. These are doubly infinite admissible sequences in the finite alphabet given by the edges of $\bar{V}$ with both possible orientations. On $\mathcal{W}\left(\Delta_{\Gamma} / \Gamma\right)$ we consider the topology generated by the sets $\mathcal{W}^{s}(\omega, \ell)=\left\{\tilde{\omega} \in \mathcal{W}\left(\Delta_{\Gamma} / \Gamma\right)\right.$ : $\left.\tilde{\omega}_{k}=\omega_{k}, k \geq \ell\right\}$ and $\mathcal{W}^{u}(\omega, \ell)=\left\{\tilde{\omega} \in \mathcal{W}\left(\Delta_{\Gamma} / \Gamma\right): \tilde{\omega}_{k}=\omega_{k}, k \leq \ell\right\}$, for $\omega \in \mathcal{W}\left(\Delta_{\Gamma} / \Gamma\right)$ and $\ell \in \mathbb{Z}$. With this topology, the space $\mathcal{W}\left(\Delta_{\Gamma} / \Gamma\right)$ is a totally disconnected compact Hausdorff space.

The invertible shift map $T$, given by $(T \omega)_{k}=\omega_{k+1}$, is a homeomorphism of $\mathcal{W}\left(\Delta_{\Gamma} / \Gamma\right)$. We can describe again the dynamical system $\left(\mathcal{W}\left(\Delta_{\Gamma} / \Gamma\right), T\right)$ in terms of subshifts of finite type.

Lemma 4.1 The space $\mathcal{W}\left(\Delta_{\Gamma} / \Gamma\right)$ with the action of the invertible shift $T$ is a subshift of finite type, where $\mathcal{W}\left(\Delta_{\Gamma} / \Gamma\right)=\mathcal{S}_{A}$ with $A$ the directed edge matrix of the finite graph $\Delta_{\Gamma} / \Gamma$.

We consider the mapping torus of $T$ :

$$
\mathcal{W}\left(\Delta_{\Gamma} / \Gamma\right)_{T}:=\mathcal{W}\left(\Delta_{\Gamma} / \Gamma\right) \times[0,1] /(T x, 0) \sim(x, 1) .
$$



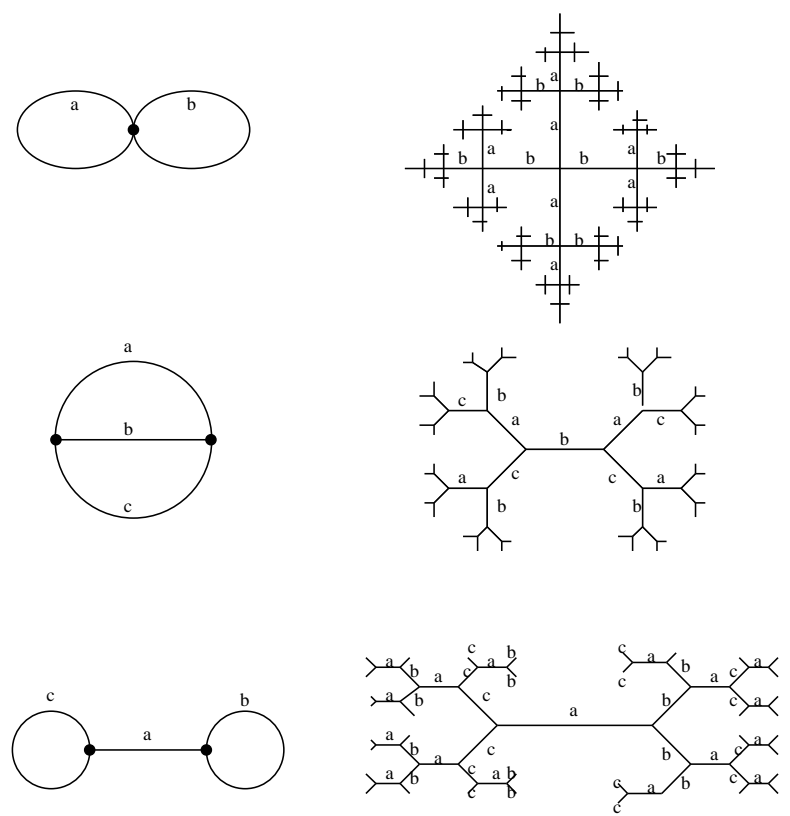

Figure 2: The graphs $\Delta_{\Gamma} / \Gamma$ for genus $g=2$, and the corresponding trees $\Delta_{\Gamma}$.

\subsection{Genus two example}

In the example of Mumford-Schottky curves of genus $g=2$, the tree $\Delta_{\Gamma}$ is illustrated in Figure 2

In the first case in the Figure, the tree $\Delta_{\Gamma}$ is just a copy of the Cayley graph of the free group $\Gamma$ on two generators, hence we can identify doubly infinite walks in $\Delta_{\Gamma}$ with doubly infinite reduced words in the generators of $\Gamma$ and their inverses. The directed edge matrix is given by

$$
A=\left(\begin{array}{llll}
1 & 1 & 0 & 1 \\
1 & 1 & 1 & 0 \\
0 & 1 & 1 & 1 \\
1 & 0 & 1 & 1
\end{array}\right) .
$$

In the second case in Figure 2 we label by $a=e_{1}, b=e_{2}$ and $c=e_{3}$ the oriented edges in the graph $\Delta_{\Gamma} / \Gamma$, so that we have a corresponding set of labels $E=\{a, b, c, \bar{a}, \bar{b}, \bar{c}\}$ for the edges in the covering $\Delta_{\Gamma}$. A choice of generators for the group $\Gamma \simeq \mathbb{Z} * \mathbb{Z}$ acting on $\Delta_{\Gamma}$ is obtained by identifying the generators $g_{1}$ and $g_{2}$ of $\Gamma$ with the chains of edges $a \bar{b}$ and $a \bar{c}$. Doubly infinite walks in the tree $\Delta_{\Gamma}$ are admissible doubly infinite sequences of such labels, where admissibility is determined by the directed edge matrix

$$
A=\left(\begin{array}{llllll}
0 & 1 & 0 & 0 & 0 & 1 \\
1 & 0 & 1 & 0 & 0 & 0 \\
0 & 1 & 0 & 1 & 0 & 0 \\
0 & 0 & 1 & 0 & 1 & 0 \\
0 & 0 & 0 & 1 & 0 & 1 \\
1 & 0 & 0 & 0 & 1 & 0
\end{array}\right)
$$

The third case in Figure 2 is analogous. A choice of generators for the group $\Gamma \simeq \mathbb{Z} * \mathbb{Z}$ acting on $\Delta_{\Gamma}$ is given by $a b \bar{a}$ and $c$. Doubly infinite walks in the tree $\Delta_{\Gamma}$ are admissible doubly infinite sequences 
in the alphabet $E=\{a, b, c, \bar{a}, \bar{b}, \bar{c}\}$, with admissibility determined by the directed edge matrix

$$
A=\left(\begin{array}{llllll}
0 & 0 & 1 & 0 & 0 & 1 \\
1 & 1 & 0 & 0 & 0 & 0 \\
0 & 0 & 1 & 1 & 0 & 0 \\
0 & 1 & 0 & 0 & 1 & 0 \\
1 & 0 & 0 & 0 & 1 & 0 \\
0 & 0 & 0 & 1 & 0 & 1
\end{array}\right) .
$$

The construction is analogous for genus $g>2$, for the various possible finite graphs $\Delta_{\Gamma} / \Gamma$. The directed edge matrix can then be written in block form as

$$
A=\left(\begin{array}{ll}
\alpha_{11} & \alpha_{12} \\
\alpha_{21} & \alpha_{22}
\end{array}\right)
$$

where each block $\alpha_{i j}$ is a \# $\#\left(\Delta_{\Gamma} / \Gamma\right)_{+}^{1} \times \#\left(\Delta_{\Gamma} / \Gamma\right)_{+}^{1}-$ matrix with $\alpha_{12}=\alpha_{12}^{t}, \alpha_{21}=\alpha_{21}^{t}$, and $\alpha_{11}=\alpha_{22}^{t}$.

\subsection{Cohomology of $\mathcal{W}(\Delta / \Gamma)_{T}$.}

Let $\Delta=\Delta_{\Gamma}$. We identify the first cohomology group $H^{1}\left(\mathcal{W}\left(\Delta_{\Gamma} / \Gamma\right)_{T}, \mathbb{Z}\right)$ with the group of homotopy classes of continuous maps of $\mathcal{W}\left(\Delta_{\Gamma} / \Gamma\right)_{T}$ to the circle. Let $\mathrm{C}\left(\mathcal{W}\left(\Delta_{\Gamma} / \Gamma\right), \mathbb{Z}\right)$ be the $\mathbb{Z}$-module of integer valued continuous functions on $\mathcal{W}\left(\Delta_{\Gamma} / \Gamma\right)$, and let

$$
\mathrm{C}\left(\mathcal{W}\left(\Delta_{\Gamma} / \Gamma\right), \mathbb{Z}\right)_{T}:=\operatorname{Coker}(\delta),
$$

for $\delta(f)=f-f \circ T$. The analog of Theorem 3.4 holds:

Proposition 4.2 The map $f \mapsto[\exp (2 \pi i t f(x))]$, which associates to an element $f \in \mathrm{C}\left(\mathcal{W}\left(\Delta_{\Gamma} / \Gamma\right), \mathbb{Z}\right)$ a homotopy class of maps from $\mathcal{W}\left(\Delta_{\Gamma} / \Gamma\right)_{T}$ to the circle, gives an isomorphism $\mathrm{C}\left(\mathcal{W}\left(\Delta_{\Gamma} / \Gamma\right), \mathbb{Z}\right)_{T} \simeq$ $H^{1}\left(\mathcal{W}\left(\Delta_{\Gamma} / \Gamma\right)_{T}, \mathbb{Z}\right)$. Moreover, there is a filtration of $\mathrm{C}\left(\mathcal{W}\left(\Delta_{\Gamma} / \Gamma\right), \mathbb{Z}\right)_{T}$ by free $\mathbb{Z}$-modules $F_{0} \subset F_{1} \subset$ $\cdots F_{n} \cdots$, of rank $\theta_{n}-\theta_{n-1}+1$, where $\theta_{n}$ is the number of admissible words of length $n+1$ in the alphabet, so that we have

$$
H^{1}\left(\mathcal{W}\left(\Delta_{\Gamma} / \Gamma\right)_{T}, \mathbb{Z}\right)=\underset{n}{\lim _{n}} F_{n}
$$

The quotients $F_{n+1} / F_{n}$ are also torsion free.

The space $\mathcal{W}\left(\Delta_{\Gamma} / \Gamma\right)_{T}$ corresponds to a space of "bounded geodesics" on the graph $\Delta_{K} / \Gamma$, where geodesics, in this setting, are just doubly infinite walks in $\Delta_{K} / \Gamma$. In particular, a closed geodesic is the image under the quotient map $\pi_{\Gamma}: \Delta_{K} \rightarrow \Delta_{K} / \Gamma$ of a doubly infinite walk in the Bruhat-Tits tree $\Delta_{K}$ with ends given by the pair $z^{+}(\gamma), z^{-}(\gamma)$ of fixed points of some element $\gamma \in \Gamma$. Similarly, a bounded geodesic is an element $\omega \in \mathcal{W}\left(\Delta_{K} / \Gamma\right)$ which is the image, under the quotient map, of a doubly infinite walk in $\Delta_{K}$ with both ends on $\Lambda_{\Gamma} \subset \mathbb{P}^{1}(K)$. This implies that a bounded geodesic is a walk of the form $\omega=\pi_{\Gamma}(\tilde{\omega})$, for some $\tilde{\omega} \in \mathcal{W}\left(\Delta_{\Gamma} / \Gamma\right)$. By construction, any such walk is an axis of $\Delta_{\Gamma}$.

Orbits of $\mathcal{W}\left(\Delta_{\Gamma} / \Gamma\right)$ under the action of the invertible shift $T$ correspond bijectively to orbits of the complement of the diagonal in $\Lambda_{\Gamma} \times \Lambda_{\Gamma}$ under the action of $\Gamma$. Thus, we see that $\mathcal{W}\left(\Delta_{\Gamma} / \Gamma\right)_{T}$ gives a geometric realization of the space of "bounded geodesics" on the graph $\Delta_{K} / \Gamma$, much as, in the case of the geometry at arithmetic infinity, we used the mapping torus of the shift $T$ as a model of the tangle of bounded geodesics in a hyperbolic handlebody.

As in the case at infinity, we can consider the Pimsner-Voiculescu exact sequence computing the $K$-theory groups of the crossed product $\mathrm{C}^{*}$-algebra $\mathrm{C}\left(\mathcal{W}\left(\Delta_{\Gamma} / \Gamma\right)\right) \rtimes_{T} \mathbb{Z}$,

$$
0 \rightarrow H^{0}\left(\mathcal{W}\left(\Delta_{\Gamma} / \Gamma\right)_{T}, \mathbb{Z}\right) \rightarrow \mathrm{C}\left(\mathcal{W}\left(\Delta_{\Gamma} / \Gamma\right), \mathbb{Z}\right) \stackrel{\delta=1-T}{\longrightarrow} \mathrm{C}\left(\mathcal{W}\left(\Delta_{\Gamma} / \Gamma\right), \mathbb{Z}\right) \rightarrow H^{1}\left(\mathcal{W}\left(\Delta_{\Gamma} / \Gamma\right)_{T}, \mathbb{Z}\right) \rightarrow 0
$$


In the corresponding sequence

$$
0 \rightarrow H^{0}\left(\mathcal{W}\left(\Delta_{\Gamma} / \Gamma\right)_{T}, \kappa\right) \rightarrow \mathcal{P} \stackrel{\delta}{\longrightarrow} \mathcal{P} \rightarrow H^{1}\left(\mathcal{W}\left(\Delta_{\Gamma} / \Gamma\right)_{T}, \kappa\right) \rightarrow 0,
$$

for the cohomology for $H^{*}\left(\mathcal{W}\left(\Delta_{\Gamma} / \Gamma\right)_{T}, \kappa\right)$, with $\kappa=\mathbb{R}$ or $\mathbb{C}$, we can take the vector space $\mathcal{P}$ obtained, as in the case at infinity, by tensoring with $\kappa$ the $\mathbb{Z}$-module $\mathcal{P} \subset \mathrm{C}\left(\mathcal{W}\left(\Delta_{\Gamma} / \Gamma\right), \mathbb{Z}\right)$ of functions of future coordinates where $\mathcal{P} \simeq \mathrm{C}\left(\mathcal{W}^{+}\left(\Delta_{\Gamma} / \Gamma\right), \mathbb{Z}\right)$. This has a filtration $\mathcal{P}=\cup_{n} \mathcal{P}_{n}$, where $\mathcal{P}_{n}$ is identified with the submodule of $\mathrm{C}\left(\mathcal{W}^{+}\left(\Delta_{\Gamma} / \Gamma\right), \mathbb{Z}\right)$ generated by characteristic functions of $\mathcal{W}^{+}\left(\Delta_{\Gamma} / \Gamma, \rho\right) \subset$ $\mathcal{W}^{+}\left(\Delta_{\Gamma} / \Gamma\right)$, where $\rho \in \mathcal{W}^{*}\left(\Delta_{\Gamma} / \Gamma\right)$ is a finite walk $\rho=w_{0} \cdots w_{n}$ of length $n+1$, and $\mathcal{W}^{+}\left(\Delta_{\Gamma} / \Gamma, \rho\right)$ is the set of infinite paths $\omega \in \mathcal{W}^{+}\left(\Delta_{\Gamma} / \Gamma\right)$, with $\omega_{k}=w_{k}$ for $0 \leq k \leq n+1$. This filtration defines the terms $F_{n}=\mathcal{P}_{n} / \delta \mathcal{P}_{n-1}$ in the filtration of the dynamical cohomology of the Mumford curve, as in Proposition 4.2 Again, we will use the same notation in the following for the free $\mathbb{Z}$-module $\mathcal{P}_{n}$ of functions of at most $n+1$ future coordinates and the vector space obtained by tensoring $\mathcal{P}_{n}$ by $\kappa$.

We obtain a Hilbert space completion of the space $\mathcal{P}$ of cochains in (4.4) by considering $\mathcal{L}=$ $L^{2}\left(\Lambda_{\Gamma}, \mu\right)$ defined with respect to the measure on $\Lambda_{\Gamma}=\partial \Delta_{\Gamma}$ given by assigning its value on the clopen set $V(v)$, given by the ends of all paths in $\Delta_{\Gamma}$ starting at a vertex $v$, to be

$$
\mu(V(v))=q^{-d(v)-1},
$$

with $q=\operatorname{card}(\mathcal{O} / \mathfrak{m})$.

In 11] $\S 4$, we showed how the mapping torus $\mathcal{S}_{T}$ of the subshift of finite type $(\mathcal{S}, T)$, associated to the limit set of the Schottky group, maps surjectively to the tangle of bounded geodesics inside the hyperbolic handlebody, through a map that resolves all the points of intersection of different geodesics. In the case of the Mumford curve, where we replace the real hyperbolic 3-space by the Bruhat-Tits building $\Delta_{K}$, the analog of the surjective map from $\mathcal{S}_{T}$ to the tangle of bounded geodesics is a map from $\mathcal{W}\left(\Delta_{\Gamma} / \Gamma\right)_{T}$ to the dual graph $\Delta_{\Gamma} / \Gamma$. Here is a description of this map.

As before, we write elements of $\mathcal{W}\left(\Delta_{\Gamma} / \Gamma\right)$ as admissible doubly infinite sequences

$$
\omega=\ldots w_{i_{-m}} \ldots w_{i_{-1}} w_{i_{0}} w_{i_{1}} \ldots w_{i_{n}} \ldots,
$$

with the $w_{i_{k}}=\left\{e_{i_{k}}, \epsilon_{i_{k}}\right\}$ oriented edges on the graph $\Delta_{\Gamma} / \Gamma$. We consider each oriented edge $w$ of normalized length one, so that it can be parameterized as $w(t)=\{e(t), \epsilon\}$, for $0 \leq t \leq 1$, with $\bar{w}(t)=\{e(1-t),-\epsilon\}$. Since $\omega \in \mathcal{S}_{A}$ is an admissible sequence of oriented edges we have $w_{i_{k}}(1)=$ $w_{i_{k+1}}(0) \in \Delta_{\Gamma}^{(0)}$.

We consider a map of the covering space $\mathcal{W}\left(\Delta_{\Gamma} / \Gamma\right) \times \mathbb{R}$ of $\mathcal{W}\left(\Delta_{\Gamma} / \Gamma\right)_{T}$ to $\left|\Delta_{\Gamma}\right|$ of the form

$$
\tilde{E}(\omega, \tau)=w_{i_{[\tau]}}(\tau-[\tau]) .
$$

Here $\left|\Delta_{\Gamma}\right|$ denotes the geometric realization of the graph. By construction, the map $\tilde{E}$ satisfies $\tilde{E}(T \omega, \tau)=\tilde{E}(\omega, \tau+1)$, hence it descends to a map $E$ of the quotient

$$
E: \mathcal{W}\left(\Delta_{\Gamma}\right)_{T} \rightarrow\left|\Delta_{\Gamma}\right|
$$

We then obtain a map to $\left|\Delta_{\Gamma} / \Gamma\right|$, by composing with the quotient map of the $\Gamma$ action, $\pi_{\Gamma}: \Delta_{\Gamma} \rightarrow$ $\Delta_{\Gamma} / \Gamma$, that is,

$$
\bar{E}:=\pi_{\Gamma} \circ E: \mathcal{W}\left(\Delta_{\Gamma}\right)_{T} \rightarrow\left|\Delta_{\Gamma} / \Gamma\right| .
$$

Thus, we obtain the following.

Proposition 4.3 The map $\bar{E}$ of (4.7) is a continuous surjection from the mapping torus $\mathcal{W}\left(\Delta_{\Gamma}\right)_{T}$ to the geometric realization $\left|\Delta_{\Gamma} / \Gamma\right|$ of the finite graph $\Delta_{\Gamma} / \Gamma$. 
The fibers of the map (4.7) are explicitly described as

$$
\bar{E}^{-1}\left(w_{i}(t)\right)=\mathcal{W}\left(\Delta_{\Gamma}\right)\left(w_{i}\right) \times\{t\} \cup \mathcal{W}\left(\Delta_{\Gamma}\right)\left(\bar{w}_{i}\right) \times\{1-t\}
$$

where $w_{i}(t)$, for $t \in[0,1]$ is a parameterized oriented edge in the graph $\Delta_{\Gamma} / \Gamma$, and $\mathcal{W}\left(\Delta_{\Gamma}\right)\left(w_{i}\right) \subset$ $\mathcal{W}\left(\Delta_{\Gamma}\right)$ consists of

$$
\mathcal{W}\left(\Delta_{\Gamma}\right)\left(w_{i}\right)=\left\{\omega \in \mathcal{W}\left(\Delta_{\Gamma}\right): w_{i_{0}}=w_{i}\right\}
$$

for $\omega=\ldots w_{i_{-m}} \ldots w_{i_{-1}} w_{i_{0}} w_{i_{1}} \ldots w_{i_{n}} \ldots$

We can also consider the construction described above, where, instead of using the tree $\Delta_{\Gamma}$, we use larger $\Gamma$-invariant trees inside the Bruhat-Tits tree $\Delta$.

The set of doubly infinite walks $\mathcal{W}\left(\Delta_{K} / \Gamma\right)$ can be identified with the set of admissible doubly infinite sequences in the oriented edges of a fundamental domain for the action of $\Gamma$. Therefore, we obtain the following identification

$$
\mathcal{W}\left(\Delta_{K} / \Gamma\right) \simeq V \times \mathcal{W}^{*}\left(\Delta_{\Gamma} / \Gamma\right) \times V \cup V \times \mathcal{W}^{+}\left(\Delta_{\Gamma} / \Gamma\right) \cup \mathcal{W}^{+}\left(\Delta_{\Gamma} / \Gamma\right) \times V \cup \mathcal{W}\left(\Delta_{\Gamma} / \Gamma\right),
$$

where we distinguish between walks that wander off from the finite graph $\Delta_{\Gamma} / \Gamma$ along one of the paths leading to an end in $V$, in one or in both directions, and those that stay confined within the finite graph $\Delta_{\Gamma} / \Gamma$. Here we include in $\mathcal{W}^{*}\left(\Delta_{\Gamma} / \Gamma\right)=\cup_{n} \mathcal{W}^{n}\left(\Delta_{\Gamma} / \Gamma\right)$ also the case $\mathcal{W}^{0}\left(\Delta_{\Gamma} / \Gamma\right)=\emptyset$, where the walk in $\Delta_{K} / \Gamma$ does not intersect the finite graph $\Delta_{\Gamma} / \Gamma$ at all.

In the topology induced by the $p$-adic norm, $\mathbb{P}^{1}(K)$ is a totally disconnected compact Hausdorff space, and so is $\mathcal{W}\left(\Delta_{K} / \Gamma\right)$ by (4.9). Again, we consider the invertible shift map $T$, which is a homeomorphism of $\mathcal{W}\left(\Delta_{K} / \Gamma\right)$, and we form the mapping torus

$$
\mathcal{W}\left(\Delta_{K} / \Gamma\right)_{T}:=\mathcal{W}\left(\Delta_{K} / \Gamma\right) \times[0,1] /(T x, 0) \sim(x, 1) .
$$

We obtain the analog of Proposition 4.2 though in the case of $\Delta_{K}$ the $\mathbb{Z}$-modules $F_{n}$ will not be finitely generated. On the other hand, we can restrict to "neighborhoods" of the tree $\Delta_{\Gamma}$ inside $\Delta_{K}$, which correspond to the reduction maps modulo powers of the maximal ideal.

In fact, in the theory of Mumford curves, it is important to consider also the reduction modulo powers $\mathfrak{m}^{n}$ of the maximal ideal $\mathfrak{m} \subset \mathcal{O}_{K}$, which provides infinitesimal neighborhoods of order $n$ of the closed fiber.

For each $n \geq 0$, we consider a subgraph $\Delta_{K, n}$ of the Bruhat-Tits tree $\Delta_{K}$ defined by setting

$$
\Delta_{K, n}^{0}:=\left\{v \in \Delta_{K}^{0}: d\left(v, \Delta_{\Gamma}^{\prime}\right) \leq n\right\},
$$

with respect to the distance (4.1), with $d\left(v, \Delta_{\Gamma}^{\prime}\right):=\inf \left\{d(v, \tilde{v}): \tilde{v} \in\left(\Delta_{\Gamma}^{\prime}\right)^{0}\right\}$, and

$$
\Delta_{K, n}^{1}:=\left\{w \in \Delta_{K}^{1}: s(w), r(w) \in \Delta_{K, n}^{0}\right\} .
$$

Thus, we have $\Delta_{K, 0}=\Delta_{\Gamma}^{\prime}$. We have $\Delta_{K}=\cup_{n} \Delta_{K, n}$.

For all $n \in \mathbb{N}$, the graph $\Delta_{K, n}$ is invariant under the action of the Schottky group $\Gamma$ on $\Delta$, and the finite graph $\Delta_{K, n} / \Gamma$ gives the dual graph of the reduction $X_{K} \otimes \mathcal{O} / \mathfrak{m}^{n+1}$. They form a directed family with inclusions $j_{n, m}: \Delta_{K, n} \hookrightarrow \Delta_{K, m}$, for all $m \geq n$, with all the inclusions compatible with the action of $\Gamma$.

In [12] we introduce the dynamical cohomology and discuss spectral geometry for the $\mathrm{C}^{*}$-algebras $\mathrm{C}^{*}\left(\Delta_{K, n} / \Gamma\right) \simeq \mathrm{C}^{*}\left(\Delta_{K, n}\right) \rtimes \Gamma$.

\subsection{Spectral triples and Mumford curves.}

We consider the Hilbert space $\mathcal{H}=\mathcal{L} \oplus \mathcal{L}$ and the operator $\mathcal{D}$ defined as

$$
\left.\mathcal{D}\right|_{\mathcal{L} \oplus 0}=-\left.\frac{2 \pi}{R \log q} \sum(n+1) \hat{\Pi}_{n} \quad \mathcal{D}\right|_{0 \oplus \mathcal{L}}=\frac{2 \pi}{R \log q} \sum n \hat{\Pi}_{n},
$$


where $\hat{\Pi}_{n}=\Pi_{n}-\Pi_{n-1}$ are the orthogonal projections associated to the filtration $\mathcal{P}_{n}$, the integer $R$ is the length of all the words representing the generators of $\Gamma$ (this can be taken to be the same for all generators, possibly after blowing up a finite number of points on the special fiber, as explained in [12]), and $q=\operatorname{card}(\mathcal{O} / \mathfrak{m})$.

The same argument used in [11 for the case at arithmetic infinity adapts to the case of Mumford curves to prove the following result. (Note: the statement below corrects an unfortunate mistake that occurred in $\S 5.4$ of [12.)

Theorem 4.4 Consider the tree $\Delta_{\Gamma}$ of the p-adic Schottky group acting on $\Delta_{K}$.

1. There is a representation of the algebra $\mathrm{C}^{*}\left(\Delta_{\Gamma} / \Gamma\right)$ by bounded linear operators on the Hilbert space $\mathcal{L}$.

2. The data $\left(\mathrm{C}^{*}\left(\Delta_{\Gamma} / \Gamma\right), \mathcal{H}, \mathcal{D}\right)$, with the algebra acting diagonally on $\mathcal{H}=\mathcal{L} \oplus \mathcal{L}$, and the Dirac operator $\mathcal{D}$ of (4.11) form a spectral triple.

In [12] we recovered arithmetic information such as the local $L$-factors of [16] from the dynamical cohomology and the data of the spectral triple of Theorem 4.4 .

Recall that, for a curve $X$ over a global field $\mathbb{K}$, assuming semi-stability at all places of bad reduction, the local Euler factor at a place $v$ has the following description ([29]):

$$
L_{v}\left(H^{1}(X), s\right)=\operatorname{det}\left(1-F r_{v}^{*} N(v)^{-s} \mid H^{1}\left(\bar{X}, \mathbb{Q}_{\ell}\right)^{I_{v}}\right)^{-1}
$$

Here $F r_{v}^{*}$ is the geometric Frobenius acting on $\ell$-adic cohomology of $\bar{X}=X \otimes \operatorname{Spec}(\overline{\mathbb{K}})$, with $\overline{\mathbb{K}}$ the algebraic closure and $\ell$ a prime with $(\ell, q)=1$, where $q$ is the cardinality of the residue field $k(v)$ at $v$. We denote by $N$ the norm map. The determinant is evaluated on the inertia invariants $H^{1}\left(\bar{X}, \mathbb{Q}_{\ell}\right)^{I_{v}}$ at $v$ (all of $H^{1}\left(\bar{X}, \mathbb{Q}_{\ell}\right)$ when $v$ is a place of good reduction).

Suppose $v$ is a place of $k(v)$-split degenerate reduction. Then the completion of $X$ at $v$ is a Mumford curve $X_{\Gamma}$. In this case, the Euler factor (4.12) takes the following form:

$$
L_{v}\left(H^{1}\left(X_{\Gamma}\right), s\right)=\left(1-q^{-s}\right)^{-g} .
$$

This is computed by the zeta regularized determinant

$$
\operatorname{det}_{\infty, \pi(\mathcal{V}), i \mathcal{D}}(s)=L_{v}\left(H^{1}\left(X_{\Gamma}\right), s\right)^{-1}
$$

where

$$
\operatorname{det}_{\infty, a, i \mathcal{D}}(s):=\exp \left(-\zeta_{a, i \mathcal{D},+}^{\prime}(s, 0)\right) \exp \left(-\zeta_{a, i \mathcal{D},-}^{\prime}(s, 0)\right)
$$

for

$$
\begin{aligned}
& \zeta_{a, i \mathcal{D},+}(s, z):=\sum_{\lambda \in \operatorname{Spec}(i \mathcal{D}) \cap i[0, \infty)} \operatorname{Tr}\left(a \Pi_{\lambda}\right)(s+\lambda)^{-z} \\
& \zeta_{a, i \mathcal{D},-}(s, z):=\sum_{\lambda \in \operatorname{Spec}(i \mathcal{D}) \cap i(-\infty, 0)} \operatorname{Tr}\left(a \Pi_{\lambda}\right)(s+\lambda)^{-z} .
\end{aligned}
$$

The element $a=\pi(\mathcal{V})$ is the projection onto a linear subspace $\mathcal{V}$ of $\mathcal{H}$, which is obtained via embeddings of the cohomology of the dual graph $\Delta_{\Gamma} / \Gamma$ into the space of cochains of the dynamical cohomology.

The projection $\pi(\mathcal{V})$ acts on the range of the spectral projections $\hat{\Pi}_{n}$ of $D$ as elements $Q_{n}$ in the AF algebra core of the $\mathrm{C}^{*}$-algebra $\mathrm{C}^{*}\left(\Delta_{\Gamma} / \Gamma\right)$.

Notice how, unlike the local factor at infinity, the factor at the non-archimedean places involves the full spectrum of $D$ and not just its positive or negative part. It is believed that this difference should correspond to the presence of an underlying geometric space based on loop geometry, which manifests itself as loops at the non-archimedean places and as "half loops" (holomorphic disks) at arithmetic infinity.

There is another important difference between the archimedean and non-archimedean cases. At the archimedean prime the local factor is described in terms of zeta functions for a Dirac operator 
$D$ (cf. 11], 15]). On the other hand, at the non-archimedean places, in order to get the correct normalization as in [17, we need to introduce a rotation of the Dirac operator by the imaginary unit, $D \mapsto i D$. This rotation corresponds to the Wick rotation that moves poles on the real line to poles on the imaginary line (zeroes for the local factor) and appears to be a manifestation of a rotation from Minkowskian to Euclidean signature $i t \mapsto t$, as already remarked by Manin (23] p.135), who wrote that "imaginary time motion" may be held responsible for the fact that zeroes of $\Gamma(s)^{-1}$ are purely real whereas the zeroes of all non-archimedean Euler factors are purely imaginary. It is expected, therefore, that a more refined construction would involve a version of spectral triples for Minkowskian signature.

\section{References}

[1] C. Antonescu, E. Christensen, Spectral triples for AF C*-algebras and metrics on the Cantor set, preprint arXiv math.OA/0309044

[2] T. Bates, D. Pask, I. Raeburn, W. Szymański, The $C^{*}$-algebras of row finite graphs, New York J. Math. 6 (2000) 307-324.

[3] P. Baum, A. Connes, Geometric K-theory for Lie groups and foliations. Preprint IHES (M/82/), 1982; l'Enseignement Mathematique, t. 46, 2000, 1-35.

[4] M. Boyle, D. Handelman, Orbit equivalence, flow equivalence, and ordered cohomology, Israel J. Math. 95 (1996) 169-210.

[5] L. Chekhov, A. Mironov, A. Zabrodin, Multiloop calculations in p-adic string theory and BruhatTits trees. Comm. Math. Phys. 125 (1989), no. 4, 675-711.

[6] A. Connes, Cyclic cohomology and the transverse fundamental class of a foliation. In: Geometric methods in operator algebras (Kyoto, 1983). Pitman Res. Notes in Math., 123, Longman, Harlow $1986,52-144$.

[7] A. Connes, Compact metric spaces, Fredholm modules, and hyperfiniteness, Ergodic Theory and Dynamical Systems 9 (1989) 207-220.

[8] A. Connes, Noncommutative geometry, Academic Press, 1994.

[9] A. Connes, Geometry from the spectral point of view. Lett. Math. Phys. 34 (1995), no. 3, 203-238.

[10] C. Consani, Double complexes and Euler L-factors, Compositio Math. 111 (1998) 323-358.

[11] C. Consani, M. Marcolli, Noncommutative geometry, dynamics and $\infty$-adic Arakelov geometry, preprint arXiv:math.AG/0205306 to appear in Selecta Mathematica.

[12] C. Consani, M. Marcolli, Spectral triples from Mumford curves, International Math. Research Notices, 36 (2003) 1945-1972.

[13] J. Cuntz, A class of $C^{*}$-algebras and topological Markov chains II: reducible chains and the Ext-functor for $C^{*}$-algebras, Invent. Math. 63 (1981) 25-40.

[14] J. Cuntz, W. Krieger, A class of $C^{*}$-algebras and topological Markov chains, Invent. Math. 56 (1980) 251-268.

[15] C. Deninger, On the $\Gamma$-factors attached to motives, Invent. Math. 104 (1991) 245-261.

[16] C. Deninger, Local L-factors of motives and regularized determinants. Invent. Math. 107 (1992), no. $1,135-150$. 
[17] C. Deninger, Motivic L-functions and regularized determinants, in "Motives", Proceedings of Symposia in Pure Mathematics, Vol. 55 (1994) Part I, 707-743.

[18] A. Kumjian, D. Pask, $C^{*}$-algebras of directed graphs and group actions, Ergod. Th. \& Dynam. Sys. (1999) 19 1503-1519.

[19] A. Kumjian, D. Pask, I. Raeburn, Cuntz-Krieger algebras of directed graphs, Pacific J. Math. 184 (1998) N.1, 161-174.

[20] Yu.I. Manin, p-adic automorphic functions. Journ. of Soviet Math., 5 (1976) 279-333.

[21] Yu.I. Manin, Cubic forms, North Holland 1974.

[22] Yu.I. Manin, Three-dimensional hyperbolic geometry as $\infty$-adic Arakelov geometry, Invent. Math. 104 (1991) 223-244.

[23] Yu.I. Manin, Lectures on zeta functions and motives, Astérisque 228 (1995) 121-163.

[24] Yu.I. Manin, M. Marcolli, Continued fractions, modular symbols, and noncommutative geometry, Selecta Mathematica, New Ser. Vol.8 N.3 (2002) 475-520.

[25] M. Marcolli, Limiting modular symbols and the Lyapunov spectrum, Journal of Number Theory, Vol.98 N.2 (2003) 348-376.

[26] D. Mumford, An analytic construction of degenerating curves over complete local rings, Compositio Math. 24 (1972) 129-174.

[27] W. Parry, S. Tuncel, Classification problems in ergodic theory, London Math. Soc. Lecture Notes Series 67, 1982.

[28] G. Robertson, Boundary actions for affine buildings and higher rank Cuntz-Krieger algebras, in J. Cuntz, S. Echterhoff (eds.) "C*-algebras", Springer Verlag 2001, pp. 182-202.

[29] J. P. Serre, Facteurs locaux des fonctions zêta des variétés algébriques (définitions et conjectures). Sém. Delange-Pisot-Poitou, exp. 19, 1969/70.

[30] J. Spielberg, Free-product groups, Cuntz-Krieger algebras, and covariant maps. Internat. J. Math. 2 (1991), no. 4, 457-476.

[31] D. Sullivan, On the ergodic theory at infinity of an arbitrary discrete group of hyperbolic motions. Riemann surfaces and related topics: Proceedings of the 1978 Stony Brook Conference (State Univ. New York, Stony Brook, N.Y., 1978), pp. 465-496, Ann. of Math. Stud., 97, Princeton Univ. Press, 1981.

Caterina Consani, Department of Mathematics, University of Toronto, Canada. email: kc@math.toronto.edu

Matilde Marcolli, Max-Planck-Institut für Mathematik, Bonn Germany. email: marcolli@mpim-bonn.mpg.de 